\title{
On the incorporation of nickel into hexagonal barium titanate: magnetic properties and electron paramagnetic resonance (EPR)
}

\author{
R. Böttcher ${ }^{1}$, H. T. Langhammer ${ }^{2, *}$ (D), T. Walther ${ }^{2}$, S. Kücker ${ }^{2}$, and S. G. Ebbinghaus ${ }^{2}$ (1) \\ ${ }^{1}$ Fakultät für Physik und Geowissenschaften, Universität Leipzig, Linnéstraße 5, 04103 Leipzig, Germany \\ ${ }^{2}$ Institut für Chemie, Martin-Luther-Universität Halle-Wittenberg, Kurt-Mothes-Straße 2, 06120 Halle, Germany
}

Received: 6 October 2020

Accepted: 13 November 2020

Published online:

10 December 2020

(C) The Author(s) 2020

\begin{abstract}
Systematic measurements of the magnetic moment of hexagonal $6 \mathrm{H}-\mathrm{BaTiO}_{3-}$ $+0.04 \mathrm{BaO}+x \mathrm{NiO}(0.005 \leq x \leq 0.02)$ ceramics were performed to study the influence of $\mathrm{Ni}$ ions on the magnetic properties. By temperature-dependent measurements of the paramagnetic susceptibility at $90 \mathrm{kOe}$, the $\mathrm{Ni}^{2+}$ ion was identified as the majority defect in air-sintered Ni-doped hexagonal barium titanate. Q-band EPR investigations of a $2.0 \mathrm{~mol} \% \mathrm{Ni}$-doped single crystal revealed three different $\mathrm{Ni}$ centers located at $\mathrm{Ti}$ sites: first, $\mathrm{Ni}^{3+}$ ions at Ti sites in intact oxygen octahedra, second, $\mathrm{Ni}^{3+}$ associated with an oxygen vacancy and third, the presence of $\mathrm{Ni}^{2+}$ centers leading to a forbidden transition. The $\mathrm{Ni}^{3+}-$ $\mathrm{V}_{\mathrm{O}}$ associate characterized by a vacancy in the face-sharing oxygen plane of the oxygen octahedra can be ruled out. The crystal field parameters of the $\mathrm{Ni}^{2+}$ defect were estimated by a combined fitting of the paramagnetic susceptibility and the EPR fine structure parameter $D$ to $B_{0}^{4} \approx-17,300 \mathrm{~cm}^{-1},\left|B_{0}^{2}\right| \approx$ $2500 \mathrm{~cm}^{-1}$ and $B_{3}^{4} \approx 19,000 \mathrm{~cm}^{-1}$.
\end{abstract}

\section{Introduction}

Barium titanate as the prototype of the large perovskite oxide family with ferroelectric and piezoelectric properties is in the focus of solid-state research since decades. Aside from the continuous materials' investigation for capacitors, actuators and other passive electronic components, its potential multiferroic properties and the fact that suitably doped $\mathrm{BaTiO}_{3}$ is a dilute magnetic oxide (DMO) [1-4] proves the importance of further research on this material. DMOs or oxide diluted magnetic semiconductors (O-DMS) are promising materials for spintronics and magneto-optics [4-6]. While most of the related investigations used iron as dopant [1-4, 7-9],

Handling Editor: Till Froemling.

Address correspondence to E-mail: hans.langhammer@physik.uni-halle.de 
previously also nickel-doped $\mathrm{BaTiO}_{3}$ was studied [9-11].

Barium titanate $\left(\mathrm{BaTiO}_{3}\right)$ has a variety of phase transitions. Besides the well-known temperaturedriven phase transitions

cubic $\stackrel{125^{\circ} \mathrm{C}}{\longrightarrow}$ tetragonal $\stackrel{0^{\circ} \mathrm{C}}{\longrightarrow}$ orthorhombic $\stackrel{-90^{\circ} \mathrm{C}}{\longrightarrow}$ rhombohedral

of the modifications with $3 \mathrm{C}$ stacking, this perovskite possesses a hexagonal high-temperature phase with $6 \mathrm{H}$ stacking, which can be stabilized down to room temperature via sintering and cooling in reducing atmosphere [12] or by doping with several 3d transition elements [13-15]. The $6 \mathrm{H}$ modification of $\mathrm{BaTiO}_{3}$ exhibits its own series of temperature-driven phase transitions [16, 17]:

hexagonal $\stackrel{-51^{\circ} \mathrm{C}}{\longrightarrow}$ orthorhombic $\stackrel{-199^{\circ} \mathrm{C}}{\longrightarrow}$ monoclinic

The hexagonal $6 \mathrm{H}$ polytype of $\mathrm{BaTiO}_{3}$ (space group $\mathrm{P}_{3} / \mathrm{mmc}$ ) has two non-equivalent $\mathrm{Ti}$ sites per unit cell with trigonal symmetry. The $\operatorname{Ti}(1)$ site refers to the $2 \mathrm{a}$ position $(0,0,0)$ belonging to exclusively corner-sharing oxygen octahedra, while $\mathrm{Ti}(2)$ refers to the $4 \mathrm{f}$ position $(1 / 3,2 / 3,0.154)$ belonging to a $\mathrm{Ti}_{2} \mathrm{O}_{9}$ unit of two face-sharing oxygen octahedra. In the following, the face-sharing oxygen ions are denoted as $\mathrm{O}(1)$ and the ones connecting the $\mathrm{Ti}_{2} \mathrm{O}_{9}$ units and the corner-sharing octahedra as $\mathrm{O}(2)$. This labeling of the atoms follows the one of Burbank and Evans [18]. The unit cell of hexagonal $6 \mathrm{H}-\mathrm{BaTiO}_{3}$ can also be described by six $\mathrm{BaO}_{3}$ layers (i.e., $\left[\mathrm{Ba}(2) \mathrm{O}(2)_{3-}\right.$ $\left.\mathrm{Ba}(2) \mathrm{O}(2)_{3} \mathrm{Ba}(1) \mathrm{O}(1)_{3}\right]_{2}$ ) forming a (cch $)_{2}$ sequence, where c corresponds to corner-sharing and $\mathrm{h}$ to facesharing layers of the $\mathrm{TiO}_{6}$ octahedra, respectively. The crystal structures of the $6 \mathrm{H}$ orthorhombic (space group $\mathrm{C} 222_{1}$ [17]) and $6 \mathrm{H}$ monoclinic phase (space group P112 [17]) exhibit the same stacking of the $\mathrm{BaO}_{3}$ layers with only small shifts of the atomic positions.

The ability of the $3 \mathrm{~d}$ transition element nickel to stabilize the hexagonal phase of $\mathrm{BaTiO}_{3}$ at room temperature (RT) was first reported by Glaister et al. [13] in 1960. Later work on Ni-doped $\mathrm{BaTiO}_{3}$ concerned its acceptor properties $[19,20]$. In that work, the valence state of $\mathrm{Ni}_{\mathrm{Ti}}$ was determined to be $2+$ by measuring the temperature-dependent magnetic susceptibility and by thermogravimetry. This oxidation state was found to be independent of the oxygen partial pressure in a wide range between $10^{-15}$ and $1.5 \cdot 10^{7} \mathrm{~Pa}$.

As a special property of $\mathrm{BaTiO}_{3}$, already small amounts of $\mathrm{Sr}$ distinctly shift the phase transition temperature cubic-hexagonal to higher temperatures [21]. Therefore, many papers reported only on 3C$\mathrm{BaTiO}_{3}$ ceramics, since their specimens contained $\mathrm{Sr}$, e.g., $1.7 \mathrm{~mol} \%$ in Ref. [20]. Desu et al. determined $\mathrm{Ni}^{2+}$ as the most probable $\mathrm{Ni}$ defect in $3 \mathrm{C}-\mathrm{BaTiO}_{3}$ by binding energy calculations [22]. Much later, two articles were published on hexagonal Ni-doped $\mathrm{BaTiO}_{3}[14,15]$, but scarce investigations on the defect chemistry-such as incorporation site and valence state of the paramagnetic $\mathrm{Ni}$ ion and its interaction with oxygen vacancies-were reported. By means of local probe measurements, like EPR, the nature of the $\mathrm{Ni}$ centers in hexagonal $\mathrm{BaTiO}_{3}$ can be studied in detail. Previous to our paper [23], no EPR investigations on Ni-doped hexagonal $\mathrm{BaTiO}_{3}$ were known, whereas for $\mathrm{Ni}$ defects in $3 \mathrm{C}-\mathrm{BaTiO}_{3} \mathrm{EPR}$ investigations revealed $\mathrm{Ni}^{+}$off-centers near the $\mathrm{Ba}^{2+}$ site [24, 25].

In Ref. [23], we investigated the incorporation of $\mathrm{Ni}$ ions in $\mathrm{BaTiO}_{3}$ samples of both the $3 \mathrm{C}$ and $6 \mathrm{H}$ modification. At that time, only ceramic samples were available; therefore, by means of EPR investigations on paramagnetic ions and defects with half-integral spin numbers $S$, only the principal values of the interaction tensors of the spin-Hamiltonian operator could be derived. As a consequence, essential information for the interpretation of the measurements and especially for the structure determination of the defects was missing. Furthermore, the EPR peaks of ions and defects with integer electron spin $S$ in ceramic samples are particularly difficult to detect. As a result of zero-field splitting, they have strongly angle-dependent lines whose edge singularities in the powder spectra are smeared out by distributions of the fine structure parameters.

Two different EPR powder spectra denoted as Z1 and $\mathrm{Z} 2$ were observed in the spectra of ceramic hexagonal $\mathrm{BaTiO}_{3}$ samples and attributed to $\mathrm{Ni}^{3+}$ ( $S=1 / 2$, low spin configuration) [23]. The nearly symmetric line $Z 1$ with isotropic $g$ tensor ( $g_{\text {iso- }}$ $=2.1766 \pm 0.0006)$ was already visible in roomtemperature spectra, and its intensity and line width are determined by the Ni concentration. Furthermore, spectrum $Z 1$ is strongly temperature dependent: In the low-temperature range ( $\mathrm{T} \leq 150 \mathrm{~K}$ ), it consists of three peaks arising from an orthorhombic g-tensor. 
In ceramic samples with $x \geq 0.01$, an additional anisotropic powder pattern (spectrum Z2) with three peaks corresponding to $g_{x}=2.44 \pm 0.01, \quad g_{y}=$ $2.39 \pm 0.01$ and $g_{z}=2.00 \pm 0.01$ was observed. In the orthorhombic phase (below $222 \mathrm{~K}$ ), the Z2 lines split, showing the occurrence of a second orthorhombic spectrum. The intensities of the respective peaks were also dependent on the $\mathrm{Ni}$ concentration. Depending on the doping level, only about a maximum of $5 \%$ of the nominal $\mathrm{Ni}$ content could be related to the spectra $Z 1$ and $Z 2$. On the other hand, no EPR spectra were detectable, which could be assigned to $\mathrm{Ni}^{2+}$ ions with electron spin $S=1$. Therefore, in Ref. [23] we concluded that the remaining incorporated $\mathrm{Ni}$ must be in the EPR-silent $\mathrm{Ni}^{4+}$ state. Measurements of the magnetic susceptibility, which were later performed on the same samples, falsified this conclusion. For this reason, we extended the EPR investigations to single-crystalline $\mathrm{Ni}$-doped hexagonal $\mathrm{BaTiO}_{3}$ samples. Measurements were taken in the Q-band $(34 \mathrm{GHz})$ expecting the detection of resonances of $\mathrm{Ni}^{2+}$ centers despite its large line widths $(\Delta \mathrm{B}>50 \mathrm{G})$.

More recent work on Ni-doped hexagonal $\mathrm{BaTiO}_{3}$ ceramics confirmed the majority of defects to be $\mathrm{Ni}^{2+}$ as determined by $\mathrm{x}$-ray photoelectron spectroscopy (XPS) $[10,26]$ or to be a mixture between $\mathrm{Ni}^{2+}$ and small amounts of $\mathrm{Ni}^{3+}$ as derived from extended $x$-ray absorption fine structure (EXAFS) measurements [27]. In the latter paper, the presence of the charge-compensating oxygen vacancy in the first coordination sphere of the $\mathrm{Ni}$ defect was excluded.

In the present paper, we extend the investigations on the effect of nickel doping on the defect properties of hexagonal barium titanate by systematic measurements of the magnetic moment in dependence on temperature in the concentration range between 0.5 and $2.0 \mathrm{~mol} \% \mathrm{Ni}$. Furthermore, we present EPR measurements on a $2.0 \mathrm{~mol} \% \mathrm{Ni}$-doped hexagonal $\mathrm{BaTiO}_{3}$ single crystal revealing a $\mathrm{Ni}^{2+}$ center and an associate between $\mathrm{Ni}^{3+}$ and a oxygen vacancy.

Theoretical investigations [28] predict the associate $\mathrm{Ni}^{2+/ 3+}{ }_{\mathrm{Ti}(2)}-\mathrm{V}_{\mathrm{O}(1)}$ to be most stable, in which the oxygen vacancy is located in the $\mathrm{O}(1)$ plane connecting the face-sharing oxygen octahedra. In contrast, our current EPR data exclude the formation of this defect, in accordance with our finding in the case of Fe-doped hexagonal $\mathrm{BaTiO}_{3}[29,30]$.

\section{Experimental procedure}

Powders with the nominal composition $\mathrm{BaTiO}_{3-}$ $+0.04 \mathrm{BaO}+x \mathrm{NiO}(0.005 \leq x \leq 0.02)$ were prepared by the conventional mixed-oxide powder technique. After mixing (agate balls, water) and calcining $\left(1100{ }^{\circ} \mathrm{C}, 2 \mathrm{~h}\right)$ of $\mathrm{BaCO}_{3}$ (Solvay, VL600, $<0.1$ mol\% Sr) and $\mathrm{TiO}_{2}$ (Merck, no. 808), NiO (Merck, p.a.) was added to the $\mathrm{BaTiO}_{3}$ powder. Then, the mixture was fine-milled (agate balls, 2-propanol) and densified to disks with a diameter of $6 \mathrm{~mm}$ and a height of approximately $3 \mathrm{~mm}$. The samples were sintered in air at temperatures of $1400{ }^{\circ} \mathrm{C}$ for one hour (heating rate $10 \mathrm{~K} / \mathrm{min}$ ). A part of the sintered samples were post-annealed in flowing $\operatorname{Ar}(5 \mathrm{~N}$, $40 \mathrm{ml} / \mathrm{min}$, oxygen partial pressure approx. 0.1 Pa) at $1200{ }^{\circ} \mathrm{C}$ for two hours. The $\mathrm{Ni}$ concentration of in the grown grains of the ceramics was measured by wavelength-dispersive $\mathrm{X}$-ray electron probe microanalysis (EPMA/WDX) using a CAMEBAX (Cameca). The WDX analysis confirmed the nominal $\mathrm{Ni}$ content within a relative uncertainty of $10 \%$.

Single crystals of nominal composition $\mathrm{BaTi}_{0.98-}$ $\mathrm{Ni}_{0.02} \mathrm{O}_{3}$ were grown starting from a powder prepared by the technique described above. Rods for the growth experiments were obtained by hydrostatic pressing followed by sintering in air. Crystal growth experiments were carried out in a Crystal Systems Corporation optical floating zone furnace model FZT-10000-H-VPO-PC equipped with four $1500 \mathrm{~W}$ halogen lamps. Nitrogen ( $5 \mathrm{~N}$ purity) with a gas flow of $100 \mathrm{ml} / \mathrm{min}$ was used as atmosphere. During the crystal growth, feed and seed rods were counter-rotated with $25 \mathrm{rpm}$. The resulting crystal boules were cut into pieces of approximately $1 \times 1 \times 1 \mathrm{~mm}^{3}$ with a diamond wheel saw. Single crystallinity of the samples was checked using a four-circle single-crystal diffractometer (STOE Stadi4, graphite monochromator) operating with $\mathrm{Mo}^{-\mathrm{K}_{\alpha}}$ radiation. This diffractometer was also used for orientation of the crystals. To describe the orientation of the crystals, we use the Cartesian laboratory co-ordinate system $x_{L}, y_{L}, z_{L}$, which is related to the hexagonal crystallographic co-ordinate system by $x_{L} \|[100]_{\mathrm{h}}(=a)$, $z_{L} \|[001]_{\mathrm{h}}(=c), y_{L}$ perpendicular to $x_{L}$ and $z_{L}$., i.e., $\|[120]_{h}$ (the index $h$ refers to the hexagonal crystal system). For EPR investigations, a sample with [210] orientation (with an accuracy $\leq 1^{\circ}$ ) was glued to a high-purity quartz rod. With this orientation, a rotation in the crystallographic bc-plane is achieved. 
Magnetic measurements were taken using the ACMS magnetometer option of a PPMS 9 (Quantum Design). The temperature-dependent magnetic moments were measured at maximum accessible field of $90 \mathrm{kOe}$ in the temperature range of 5-300 K using field-cooled (FC) and zero-field-cooled (ZFC) conditions. The obtained magnetic moments were corrected for the magnetic moment of the sample holder and with respect to the magnetic contribution of the $\mathrm{BaTiO}_{3}$ matrix, which was determined from undoped samples.

Single-crystal EPR measurements were taken with a Bruker EMX spectrometer (Q-band 34.0 GHz) with $100 \mathrm{kHz}$ field modulation using a cylindrical cavity ER 5106 QT $\left(\mathrm{TE}_{012}\right)$ in connection with an Oxford Instruments cryostat CF 935. The microwave frequencies were determined by an electronic frequency counter. The angular dependences of the resonance fields were measured by rotating the single crystals in steps of $5^{\circ}$ around the $[210]_{h}$-axis.

Both the spectra of the single crystal and their angular dependences were evaluated using the MATLAB ${ }^{1}$ toolbox for electron paramagnetic resonance 'EasySpin 5.2.27' and the toolbox 'Optimization' [31]. For evaluating the spectral parameters of the Ni defects, the spin Hamiltonian

$\hat{H}=\mu_{B} \vec{B} g \hat{\vec{S}}$

and

$\hat{H}=\mu_{B} \vec{B} \underset{=}{g} \hat{\vec{S}}+D\left[\hat{\vec{S}}_{z}^{2}-\frac{1}{3} S(S+1)\right]$

are used for spin-1/2 $\left(\mathrm{Ni}^{3+}\right)$ and spin-1 $\left(\mathrm{Ni}^{2+}\right)$ centers, respectively [32] ( $\mu_{B}$ is Bohr's magneton, $\vec{B}$ the static magnetic field, $g$ the g-tensor, $\hat{\vec{S}}$ the electron spin operator, D the axial fine structure parameter). The characteristics of single-crystal EPR spectra (number of lines, angular dependence of the resonance fields and their intensities) of $\mathrm{Ni}^{2+}$ ions with the electron spin $S=1$ are essentially determined by the size of the zero-field splitting (ZFS; equals D in the case of $S=1$ ) compared to the energy of the microwave photons used and by the direction of the static magnetic field with respect to the crystallographic axes. There are basically three different resonances, which are assigned to the two allowed

${ }^{1}$ MATLAB is a registered trademark of the MathWorks, Inc., Natick, MA, USA. transitions $\left(\Delta M_{S}= \pm 1\right)$ and the forbidden one $\left(\Delta M_{S}= \pm 2\right)$. In the case $|D|<h f$ (h is Planck's constant and $f$ the frequency of the microwave field), all three resonances can be observed. The intensity of the line belonging to the forbidden transition shows a strong angular dependence and vanishes if $\vec{B}$ is parallel to the symmetry axis in the case of an axial ZFS. If $|D|>$ hf only the forbidden transition can be excited, so that only one single line with strong angledependent intensity appears for certain orientations of $\vec{B}$ in the spectrum (see "Spectra related to $\mathrm{Ni}^{2+\text { " }}$ section).

\section{Results and discussion}

\section{Magnetic susceptibility of ceramic samples}

The temperature-dependent molar magnetic susceptibilities $\chi_{\mathrm{mol}}$ of the samples in the concentration range between 0.5 and $2.0 \mathrm{~mol} \%$ are shown in Fig. 1 . All samples (as-sintered in air and post-annealed in Ar) were measured in a magnetic field of $90 \mathrm{kOe}$. The obtained data were normalized to the nominal $\mathrm{Ni}$ content $(x)$. Remarkably, the susceptibility of the airsintered samples with a lower Ni content, namely of 0.5 and $1.0 \mathrm{~mol} \%$, exhibits identical values, whereas the susceptibilities of the samples with an increased Ni content of 1.5 and $2.0 \mathrm{~mol} \%$ decrease with rising $\mathrm{Ni}$ content. This behavior is similar to the case of Fedoped hexagonal $\mathrm{BaTiO}_{3}$, where it was explained by an antiferromagnetic (AFM) interaction of paramagnetic (PM) $\mathrm{Fe}^{3+}$ ions at Ti sites [30]. Hence, we also

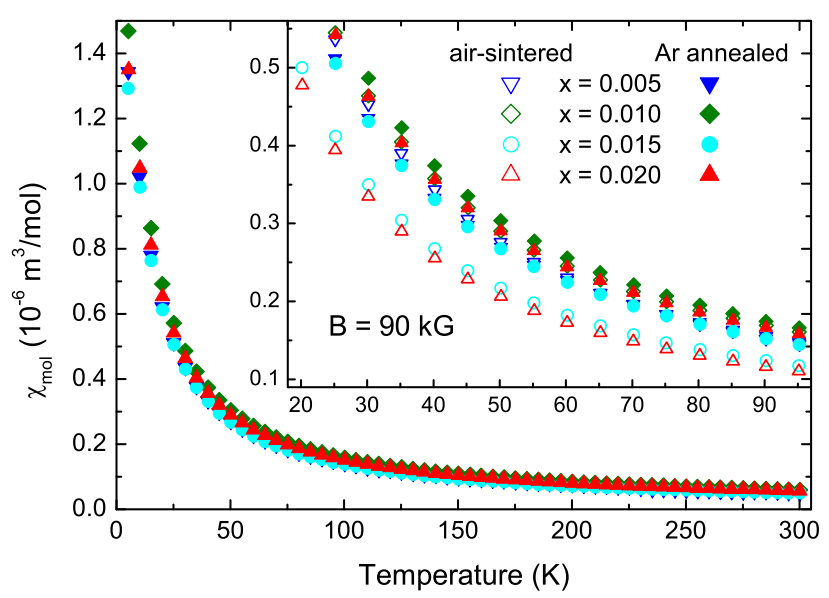

Figure 1 Temperature dependence of the molar magnetic susceptibility of Ni-doped hexagonal $\mathrm{BaTiO}_{3}$ ceramics. The insert shows an enlargement of the low-temperature region. 
assume an AFM interaction between paramagnetic $\mathrm{Ni}$ ions reducing the magnetic susceptibility at higher $\mathrm{Ni}$ concentrations. In our previous work [23], we found by EPR measurements that only about $5 \%$ of the nominal $\mathrm{Ni}$ content of the samples forms $\mathrm{Ni}^{3+}$ ions and we proposed that the remaining nickel ions are in the $4+$ valence state, since no $\mathrm{Ni}^{2+}$ resonances could be detected in the ceramic samples. The following analysis of the susceptibility data shows that this assumption is most likely not valid and the remaining $\mathrm{Ni}$ is in the $2+$ valence state.

By annealing the samples in reducing Ar atmosphere, the concentration of $\mathrm{Ni}^{3+}$ ions is decreased to about 3\% of its value before annealing [23], thus transforming practically all $\mathrm{Ni}$ into the $2+$ valence state. Therefore, only the data of the Ar-annealed samples are evaluated in the following. The molar magnetic susceptibilities of all annealed samples in the entire concentration range from 0.5 to $2.0 \mathrm{~mol} \%$ are very similar within the measurement accuracy. The fact that also the samples with a higher Ni content exhibit the same susceptibility (in contrast to the air-sintered counterparts) can be explained by the distinct increase in the concentration of oxygen vacancies due to the treatment in reducing atmosphere (with an oxygen partial pressure of about $0.1 \mathrm{~Pa}$ ). The AFM interaction reducing the susceptibility of the air-sintered samples $(x=0.015,0.020)$ is caused by superexchange via bridging oxygen ions [30]. Hence, the decreased concentration of oxygen ions upon Ar annealing reduces the AFM interaction.

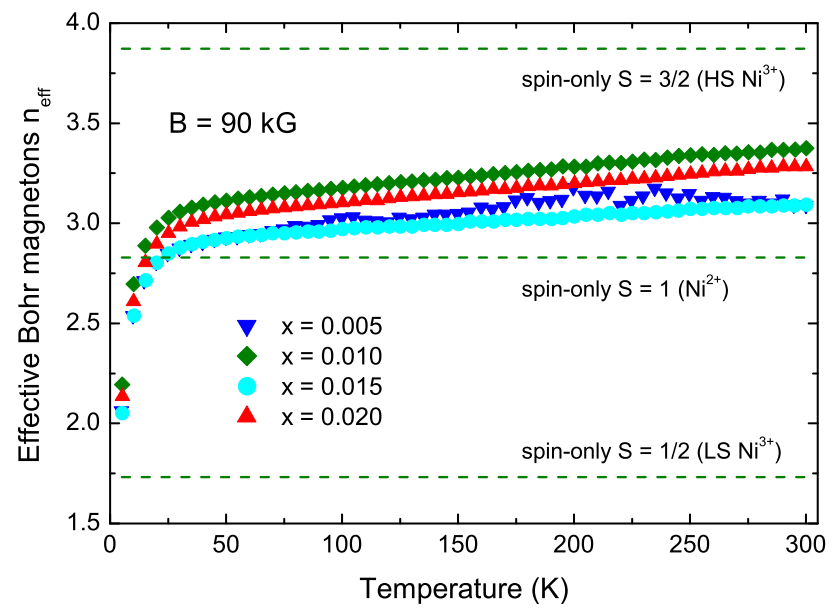

Figure 2 Effective Bohr magneton number in dependence on temperature for Ar-annealed Ni-doped hexagonal $\mathrm{BaTiO}_{3}$ ceramics. The spin-only values for $S=3 / 2, S=1$ and $S=1 / 2$ are shown by straight dashed lines.
The $n_{\text {eff }}(T)$ plot $[33,34]$ for all annealed samples is shown in Fig. 2. The effective number $n_{\text {eff }}$ of Bohr magnetons $\mu_{B}$ of the paramagnetic ion is given as

$n_{\mathrm{eff}}=\sqrt{\frac{3 k_{B} \chi_{\mathrm{mol}} T}{\mu_{0} N_{A} \mu_{B}^{2}}}$

( $k_{B}$-Boltzmann constant, $\mu_{0}$-permeability of vacuum, $N_{A}$-Avogadro constant).

Comparing the $n_{\text {eff }}$ data with the spin-only values for $S=1 / 2, S=1$ and $S=3 / 2$ clearly show the prevailing presence of $\mathrm{Ni}^{2+}$ ions $(S=1)$ in the annealed (and also as-sintered) samples. The deviation from the spin-only value is caused by the ground term $A_{2 \mathrm{~g}}$ (cubic crystal field, point group $O_{\mathrm{h}}$ ) or $A_{2}$ (trigonal crystal field, point group $\left.C_{3 \mathrm{v}}\right)$ of the $\mathrm{d}^{8}\left(\mathrm{Ni}^{2+}\right)$ configuration. The spin-only formula is a good approximation only for the $\mathrm{d}^{5}$ configuration (e.g., $\mathrm{Fe}^{3+}$ ) with ground term $A_{1 \mathrm{~g}}$ or $A_{1}$ [33]. For ground states $A_{2 \mathrm{~g}}$ and $A_{2}$, the paramagnetic susceptibility is characterized by an additional temperature-independent term causing the deviation from the spin-only formula.

A further proof of $\mathrm{Ni}^{2+}$ ions as the majority defect species both in air-sintered and in Ar post-annealed samples is the fitting of $\chi_{\mathrm{mol}}$ by the program CONCORD [35] using the ligand field approach. Details of the program can be found, for example, in [36]. A successful fit of the molar susceptibility was only possible assuming the formation of $\mathrm{Ni}^{2+}$ ions, as shown in Fig. 3. The experimental data cannot be

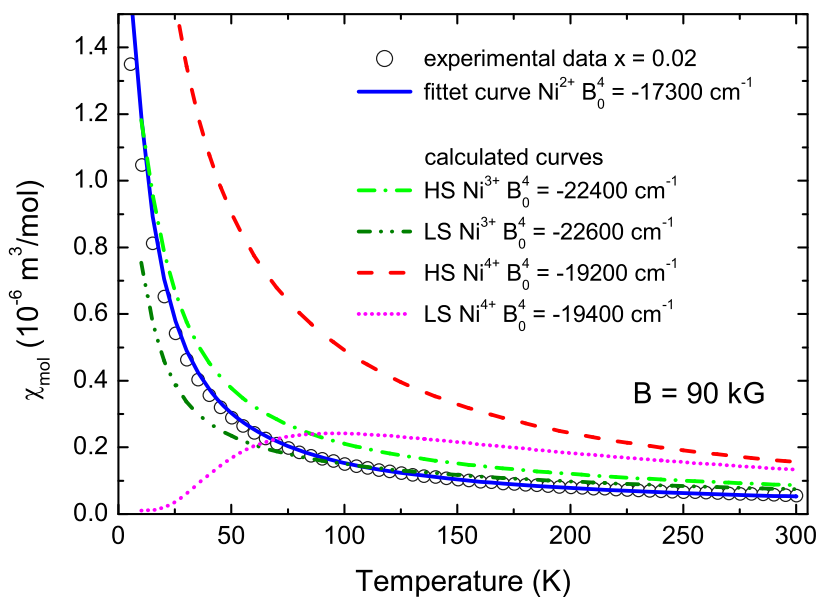

Figure 3 Experimental molar magnetic susceptibility for a $2.0 \mathrm{~mol} \%$ Ni-doped hexagonal $\mathrm{BaTiO}_{3}$ ceramics together with the fit assuming $\mathrm{Ni}^{2+}$ and calculated curves (HS/LS Ni${ }^{3+}$, HS/LS $\mathrm{Ni}^{4+}$ ) assuming a cubic crystal field. The negative sign of $B_{0}^{4}$ corresponds to the co-ordinate system with hexagonal $c$-axis as $z$. See text for details. 
described reasonably with $\mathrm{Ni}^{3+}$ or $\mathrm{Ni}^{4+}$ ions, which is demonstrated by applying different values for the octahedral crystal field parameter $B_{0}^{4}$ (Wybourne notation [37]) as also shown in Fig. 3. The $B_{0}^{4}$ values for the calculation by CONCORD are taken near the transition between high spin and low spin behavior. In hexagonal $\mathrm{BaTiO}_{3}$, the local symmetry of the oxygen octahedra at the $\operatorname{Ti}(1)$ site is $\mathrm{D}_{3 \mathrm{~d}}$ and at the $\operatorname{Ti}(2)$ site it is $C_{3 v}$. Therefore, the calculations should better be performed in the framework of a trigonal crystal field. On the other hand, the influence of the additional trigonal crystal field component (parameters $B_{0}^{2}, B_{3}^{4}$ ) on the molar susceptibility is so weak that an adequate fitting is possible already by a cubic crystal field with $B_{0}^{4}=-17,300 \mathrm{~cm}^{-1}$ (co-ordinate system with hexagonal $c$-axis as $z$-axis). As described in "Spectra related to $\mathrm{Ni}^{2+}$ " section below, the addition of further experimental data from EPR measurements is needed for the estimation of the trigonal parameters.

\section{Q-band EPR investigations on single crystals}

\section{Spectra related to $\mathrm{Ni}^{3+}$}

Figure 4a shows the Q-band EPR spectrum of the Nidoped crystal with the static magnetic field B parallel to the hexagonal c-axis. Since the single-crystalline sample was grown under reducing atmosphere (see "Experimental procedure" section) strongly decreasing the concentration of $\mathrm{Ni}^{3+}$ [23], the intensity of the $\mathrm{Ni}^{3+}$ lines is comparatively low. Dominant in this spectrum are the fine structure lines of the isolated $\mathrm{Fe}^{3+}$ ions, which are present in our crystals as an impurity of the starting materials. These lines are very sharp because of the very low Fe concentration of $\leq 70 \mathrm{ppm}$ [29]. The spectrum of the $\mathrm{Fe}-\mathrm{V}_{\mathrm{O}}$ associate [29] was also detectable but lies outside the magnetic field range shown. The presence of further paramagnetic impurities $(\mathrm{Mn}, \mathrm{Cr}$ ) leads to the resonances near the central fine structure line of the isolated $\mathrm{Fe}^{3+}$ ions.

The line of the $\mathrm{Ni}^{3+}$ center (Z1), already found in our investigations of ceramic samples [23], can easily be identified in the single-crystal spectrum due to its g-factor. The correspondence with the g-factor of spectrum $Z 1$ of the ceramics concerns both its absolute value and its symmetry at RT, which is isotropic for the ceramics [23]. Accordingly, the resonance field

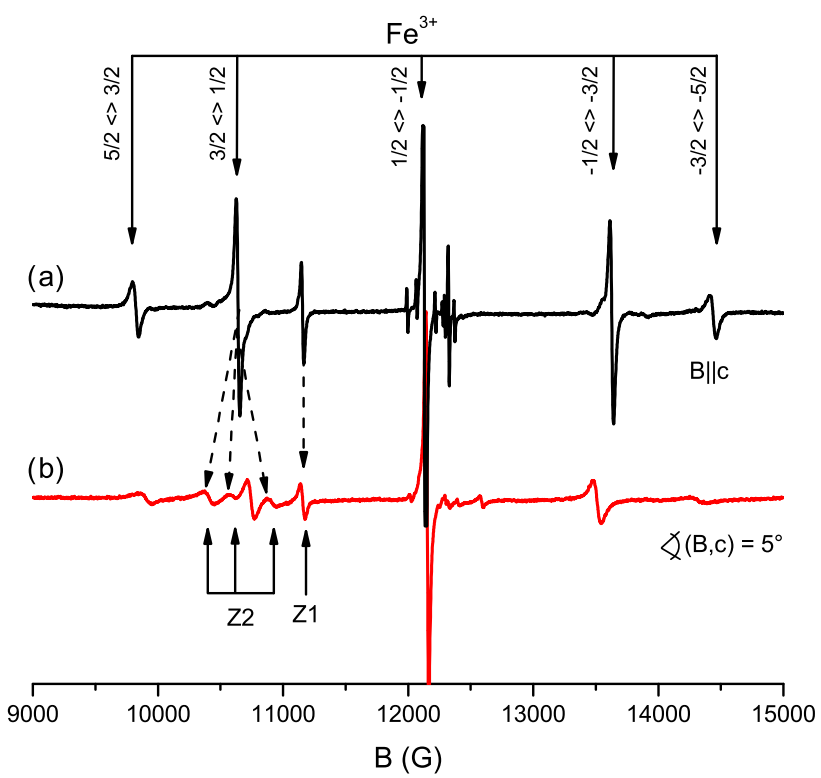

Figure 4 Room-temperature spectra of a Ni-doped hexagonal $\mathrm{BaTiO}_{3}$ single crystal measured in Q-band $(34.0 \mathrm{GHz})$ with the magnetic field $\mathrm{B}$ parallel to the hexagonal c-axis (a) and tilted by an angle of $5^{\circ}(\mathbf{b})$.

of the spectrum $Z 1$ is angle independent when the crystal is rotated around the $\left[\begin{array}{lll}2 & 1 & 0\end{array}\right]_{\mathrm{h}}$ axis (see Fig. 5). According to our previous work [23], spectrum Z1 is caused by $\mathrm{Ni}^{3+}$ surrounded by a complete oxygen octahedron without vacancy and located either on $\mathrm{Ti}(1)$ or on $\mathrm{Ti}(2)$ site. Its g-value is given in Table 1.

A second $\mathrm{Ni}^{3+}$ related spectrum, denoted as $\mathrm{Z2}$, also consists of only one line for B\|c. It overlaps with

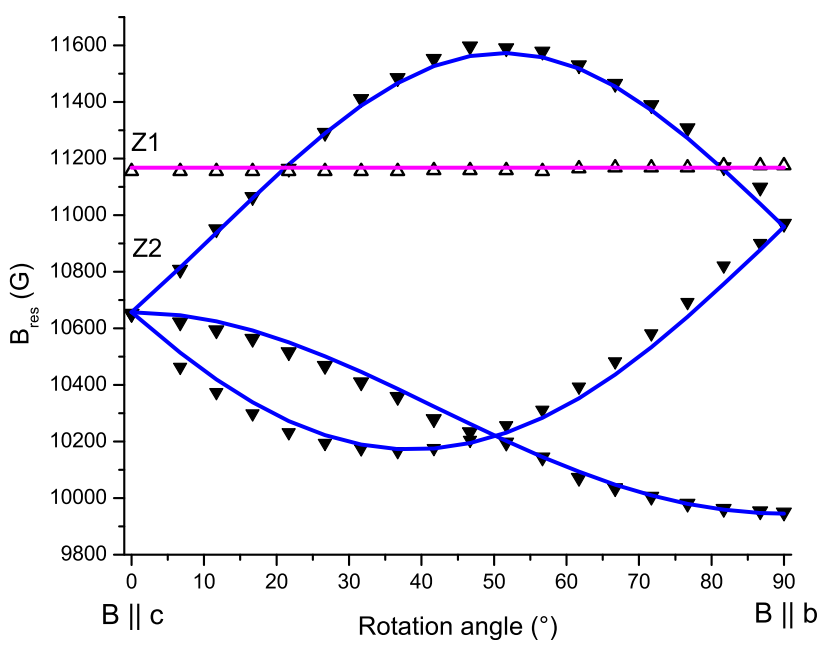

Figure 5 Angular dependence of the resonance fields of the $\mathrm{Ni}^{3+}$ centers $Z 1$ and Z2. Rotation axis is the $\left[\begin{array}{lll}2 & 1 & 0\end{array}\right]_{\mathrm{h}}$ axis. Symbols are experimental measuring points, while the curves are calculated values using the spin Hamiltonian parameters given in Table 1. 
Table 1 Spin-Hamiltonian parameters of $\mathrm{Ni}$ centers in hexagonal $6 \mathrm{H}-\mathrm{BaTiO}_{3}$ single crystals evaluated from room-temperature Q-band spectra. $\Theta$ is the angle between the $g_{z}$ eigenvector and the hexagonal c-axis

\begin{tabular}{lllllll}
\hline & $S$ & $g_{x}$ & $g_{y}$ & $g_{z}$ & $D\left(\mathrm{~cm}^{-1}\right)$ & $\Theta\left({ }^{\circ}\right)$ \\
\hline $\mathrm{Ni}^{3+} \mathrm{Z1}$ & $1 / 2$ & $2.1757(3)$ & $=g_{x}$ & $=g_{x}$ & - & 0 \\
$\mathrm{Ni}^{3+} \mathrm{Z} 2$ & $1 / 2$ & $2.4430(3)$ & $2.3829(3)$ & $2.0208(3)$ & - & $56.7(5)$ \\
$\mathrm{Ni}^{2+}$ & 1 & $2.20(1)$ & $=g_{x}$ & $=g_{x}$ & $3.55(5)$ & 0 \\
\hline
\end{tabular}

the fine structure line $M_{S}=3 / 2 \leftrightarrow M_{S}=1 / 2$ of the $\mathrm{Fe}^{3+}$ spectrum and is therefore barely visible. If the direction of the static magnetic field deviates from the hexagonal $c$-direction, this spectrum becomes clearly visible and consists of an angle-dependent triplet (Fig. 4b). Also spectrum Z2 was already detected in ceramic samples. Like $\mathrm{Z} 1$ it was assigned to $\mathrm{Ni}^{3+}$ ions possessing low-spin configuration with $S=1 / 2\left(3 \mathrm{~d}^{7}\right.$, strong octahedral field approximation). These ions are located at the other $\mathrm{Ti}$ site compared to the $\mathrm{Ni}^{3+}$ ions causing $Z 1$ [23]. The angle dependence of spectrum $Z 2$ found for the single crystal (see Fig. 5) now reveals that the previous assumption concerning the location within an intact oxygen octahedron is wrong. A qualitatively analogous angular dependence was already observed in the case of iron-doped hexagonal $\mathrm{BaTiO}_{3}$ caused by an associate between $\mathrm{Fe}^{3+}$ and a vacancy in the surrounding oxygen octahedron (see Fig. 5 in [29]). The symmetry of the angular dependence can easily be understood by the six possible positions of the associate in each $\mathrm{Ti}(1) \mathrm{O}_{6}$ or $\mathrm{Ti}(2) \mathrm{O}_{6}$ unit. For B $\|$ c, all six associate positions are equivalent, whereas for $B \| b=\left[\begin{array}{lll}0 & 1 & 0\end{array}\right]_{h}$ four positions cause a resonance field of $10.96 \mathrm{kG}$ and the remaining two positions a resonance field of $9.94 \mathrm{kG}$, which is illustrated in Fig. 9 of [29] by a sketch of the $\mathrm{TiO}_{6}$ unit in relation to the rotation axis $\left[\begin{array}{lll}2 & 1 & 0\end{array}\right]_{\mathrm{h}}$. This scenario of a $\mathrm{Ni}^{3+}-\mathrm{V}_{\mathrm{O}}$ associate is confirmed by the simulation/fitting of the angular dependencies shown in Fig. 5, resulting in the principal g-values and the directions of the principal axes of the g-tensors. The principal axis system for one of the six possible Z2 defect positions corresponds to a rotation of the Cartesian laboratory coordinate system $\mathrm{x}_{\mathrm{L}}, \mathrm{y}_{\mathrm{L}}$, $\mathrm{z}_{\mathrm{L}}$ around the axis $\mathrm{x}_{\mathrm{L}}$ by the angle $56.7^{\circ}$. The principal axis systems of the other five hexagonal symmetryrelated positions are created by rotation around the hexagonal c-axis (rotation angle $120^{\circ}$ and $240^{\circ}$ ) and reflection on the $\mathrm{x}_{\mathrm{L}}-\mathrm{y}_{\mathrm{L}}$-plane. The principal values of the orthorhombic g-tensor for the $\mathrm{Ni}^{3+}$ center $\mathrm{Z} 2$ are given in Table 1. Since its principal axis systems are tilted against the hexagonal c-axis by $56.7^{\circ}$ (respectively, $123.3^{\circ}$ as the supplementary angle), the $\mathrm{Ni}^{3+}$ ion cannot be surrounded by an intact oxygen octahedron with trigonal symmetry. In this case, one would expect an axial g-tensor with the $c$-axis as the trigonal axis. Hence, the $\mathrm{Ni}^{3+}$ center is associated with a next neighbor oxygen vacancy $\left(\mathrm{V}_{\mathrm{O}}\right)$ strongly disturbing the local symmetry at the $\mathrm{Ni}^{3+}$ site. The missing oxygen ion induces a very strong axial field, which symmetry axis lies in the $\mathrm{Ni}^{3+}-\mathrm{V}_{\mathrm{O}}$ direction. But the global hexagonal lattice symmetry reduces the local symmetry at the $\mathrm{Ni}^{3+}$ ion to an orthorhombic one, which differs only slightly from the axial symmetry. Due to the two non-equivalent Ti sites in the hexagonal $\mathrm{BaTiO}_{3}$ lattice, there are several possibilities for the formation of such associate centers, which were also detected in Fe-doped hexagonal $\mathrm{BaTiO}_{3}[29,30]$. If the exclusively corner-sharing octahedron is affected, the oxygen vacancy can be located at each of the six equivalent $\mathrm{O}(2)$ sites surrounding the $\mathrm{Ti}(1)$. Because the $\mathrm{Ni}^{3+}{ }_{\mathrm{Ti}(1)}-\mathrm{V}_{\mathrm{O}(2)}$ distances are equal in the octahedron (and therefore also the total strength of the electrical field at the $\mathrm{Ni}$ site in the differently oriented associate centers), only one kind of associate centers (type 1) is expected. Because of the six possible orientations of the $\mathrm{Ni}^{3+}{ }_{\mathrm{Ti}(1)}-\mathrm{V}_{\mathrm{O}(2)}$ associates in the crystal, type 1 generates generally six sets of EPR spectra in single-crystal experiments, which number, however, is reduced to three by the crystal symmetry.

A possible location of the $\mathrm{Ni}^{3+}-\mathrm{V}_{\mathrm{O}}$ associate in the face-sharing octahedra causes a more complex situation. In this case, two different $\mathrm{Ti}-\mathrm{O}$ distances exist due to the local $3 \mathrm{~m}$ symmetry of the $4 \mathrm{f}$ position: three shorter distances $\mathrm{R}_{\mathrm{Ti}(2)-\mathrm{O}(2)}=1.958 \AA$ and three longer ones $\mathrm{R}_{\mathrm{Ti}(2)-\mathrm{O}(1)}=1.992 \AA^{2}{ }^{2}$ Therefore, two kinds of $\mathrm{Ni}^{3+}-\mathrm{V}_{\mathrm{O}}$ associates (type 2 and 3) are expected and the g-tensors for the two types should

\footnotetext{
$\overline{{ }^{2} \text { Data from }}$ undoped $6 \mathrm{H}-\mathrm{BaTiO}_{3}$ [38].
} 
Table 2 Number $N$, length $R$ and angle $\Theta$ of the Ti-O bonds in the $\mathrm{Ti}(1) \mathrm{O}(2)_{6}$ and $\mathrm{Ti}(2)_{2} \mathrm{O}(1)_{3} \mathrm{O}(2)_{6}$ units for undoped hexagonal $6 \mathrm{H}-\mathrm{BaTiO}_{3}$ [38]. $\Theta$ is the angle between Ti-O directions and the hexagonal $c$-axis $\left(z_{L}\right)$

\begin{tabular}{llll}
\hline & $N$ & $R(\AA)$ & $\Theta\left({ }^{\circ}\right)$ \\
\hline $\operatorname{Ti}(1)-\mathrm{O}(2)$ & 6 & 1.983 & 55.62 \\
$\operatorname{Ti}(2)-\mathrm{O}(1)$ & 3 & 1.992 & 47.51 \\
$\operatorname{Ti}(2)-\mathrm{O}(2)$ & 3 & 1.958 & 58.40 \\
\hline
\end{tabular}

differ. The EPR spectra of type 2 and 3 have the same symmetry properties with respect to the rotation pattern as those of type 1 . If structurally different $\mathrm{Ni}^{3+}-\mathrm{V}_{\mathrm{O}}$ centers (type $1-3$ ) possess almost equal gtensors, the line separation of different spectral types should be easier detected in the Q-band than in the Xband spectra. Unfortunately, in the Q-band spectra of the Z2 center, no splitting (average line width about $50 \mathrm{G})$ was observed. From this finding, it can be concluded that either the differences in the principal values of the g-tensors are smaller than $\Delta g=5 \cdot 10^{-2}$ or there is only one type of $\mathrm{Ni}^{3+}-\mathrm{V}_{\mathrm{O}}$ associate.

Since the principal g-values of Z2 differ only slightly from axial symmetry (Table 1), it can be concluded that the strength of the trigonal component of the local crystal field is smaller than the field induced by the formation of the oxygen vacancy. Hence, the total field is nearly axial symmetric with the direction of one of the Ti-O bonds as its symmetry axis. This direction corresponds to the principal axis of the eigenvalue 2.02 of the $g$-tensor, since the other two eigenvalues are approximately identical and different from this value. The corresponding angle of the $\mathrm{Ti}(1)-\mathrm{O}(2)$ bond directions in the $\mathrm{Ti}(1) \mathrm{O}(2)_{6}$ units and that of the $\mathrm{Ti}(2)-\mathrm{O}(2)$ bond directions in the $\operatorname{Ti}(2) \mathrm{O}(2)_{3}$ units is $55.6^{\circ}$ and $58.4^{\circ}$, respectively (Table 2 ). Both values differ only slightly from the experimentally observed value of $56.7^{\circ}$. We therefore conclude that the $\mathrm{Ni}^{3+}-\mathrm{V}_{\mathrm{O}}$ associate is formed either as $N i_{T i(1)}^{3+}-V_{O(2)}$ or as $N i_{T i(2)}^{3+}-V_{O(2)}$. The third possibility $\mathrm{Ni}_{\mathrm{Ti}(2)}^{3+}-V_{O(1)}$ can be ruled out as it would lead to an angle of $47.5^{\circ}$ (Table 2). In that case, the oxygen vacancy would be located in the $\mathrm{O}(1)$ plane between the $\mathrm{Ti}(2)$ sites of the $\mathrm{Ti}_{2} \mathrm{O}_{9}$ units. This finding completely corresponds to our investigations in hexagonal Fe-doped $\mathrm{BaTiO}_{3}$ also detecting only one $\mathrm{Fe}^{3+}-\mathrm{V}_{\mathrm{O}}$ associate and proposing the same possible locations as in the present work $[29,30]$.
Spectra related to $\mathrm{Ni}^{2+}$

Besides the spectra discussed so far, an additional broad line was observed in the range 5800-17,500 G. Systematic measurements in the entire field range of the Q-band spectrometer showed that this signal vanishes for B $\|$ c. Its resonance field, line width and intensity are strongly dependent on the rotation angle around the $\left[\begin{array}{lll}2 & 1 & 0\end{array}\right]_{\mathrm{h}}$ axis. In Fig. 6, room-temperature spectra for different orientations are shown, and in Fig. 7 the corresponding resonance fields are depicted as a function of the rotation angle. Because our Qband spectrometer has a maximum static magnetic field strength of $18 \mathrm{kG}$, the resonance fields could not be determined for rotation angles above $75^{\circ}$. Due to the observed angle dependence, we assign $\mathrm{Ni}^{2+}$ ions $(S=1)$ to this spectrum and describe it by an axial spin Hamiltonian operator (2). The fitting of the experimental angular dependence of the resonance field $B_{\text {res }}$ resulted in the spin Hamiltonian parameters $g=2.20$ and $D=3.55 \mathrm{~cm}^{-1}$ (Table 1). The expected anisotropy of the $g$-tensor could not be resolved because of the rather high line width. The zero-field splitting (equals $D$ in the case of $S=1$ ) of $3.55 \mathrm{~cm}^{-1}$ is greater than the Q-band microwave photon energy of $1.134 \mathrm{~cm}^{-1}(f=34.0 \mathrm{GHz})$. In turn the allowed transitions $\Delta M_{S}= \pm 1$ cannot be detected with our Qband spectrometers. This situation is illustrated in Fig. 8 showing the magnetic field dependence of the three lowest electron energy eigenvalues $\left(M_{S}=-1\right.$, $0,+1$ ) calculated by CONCORD [35] for a ZFS of $3.55 \mathrm{~cm}^{-1}$, an angle of $20^{\circ}$ between $\vec{B}$ and $c$ (corresponding to the black line EPR spectrum in Fig. 6) and for two different values for $B_{0}^{2}$. The sign of $B_{0}^{2}$ corresponds to the kind of trigonal distortion of the oxygen octahedron. $B_{0}^{2}>0$ represents an elongation in c-direction, which is the case for the $\operatorname{Ti}(2)$ site in undoped $\mathrm{BaTiO}_{3}$, whereas the compressed octahedron at the $\mathrm{Ti}(1)$ site leads to $B_{0}^{2}<0$. In both cases, the Q-band photon energy of $1.134 \mathrm{~cm}^{-1}$ cannot induce allowed transitions. Only the forbidden transition $\mathrm{M}_{\mathrm{S}}=-1 \leftrightarrow+1$ can be observed and identified by its peculiarities, namely the very strong angle dependence of the line width and intensity. Due to the strong angle dependence of the magnetic resonance field in connection with the rather weak signal and the large line width, it was not possible to identify this peak in our previous EPR investigation on ceramic samples, leading to a wrong interpretation of the 


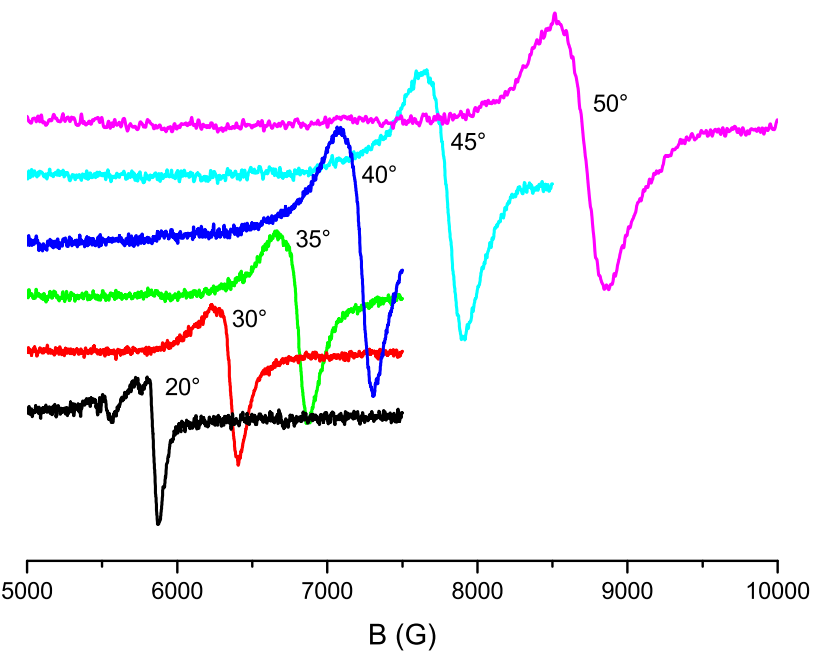

Figure 6 Selected experimental $\mathrm{Ni}^{2+} \mathrm{Q}$-band spectra for the $\mathrm{Ni}$ doped hexagonal $6 \mathrm{H} \mathrm{BaTiO}_{3}$ single crystal at RT. The spectra are marked by the rotation angle between the magnetic field $\mathrm{B}$ and the hexagonal c-axis with crystal rotation around the $\left[\begin{array}{lll}2 & 1 & 0\end{array}\right]_{\mathrm{h}}$ direction.

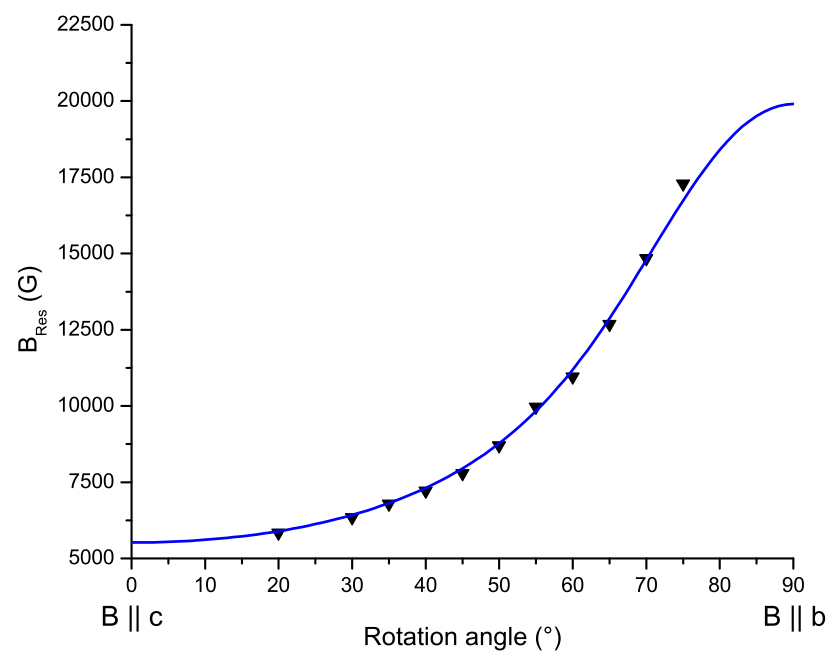

Figure 7 Angular dependence of the experimental and calculated resonance fields of the $\mathrm{Ni}^{2+}$ centers in a hexagonal $6 \mathrm{H}-\mathrm{BaTiO}_{3}$ single crystal ( $Q$-band, RT). Symbols denote experimental data and the curves are calculated values using the spin Hamiltonian parameters given in Table 1 . Rotation axis is the $\left[\begin{array}{lll}2 & 1 & 0\end{array}\right]_{\mathrm{h}}$ axis.

valence state of the majority $\mathrm{Ni}$ defects in the hexagonal $\mathrm{BaTiO}_{3}$ [23]. The current angle-dependent single-crystal measurements, on the other hand, clearly reveal this forbidden transition. Since the sign of $\mathrm{D}$ cannot be determined by EPR experiments at room temperature, the incorporation site of $\mathrm{Ni}^{2+}$, $\mathrm{Ti}(1)$ or $\mathrm{Ti}(2)$, remains unclear for the following reasons: It is known from our EPR investigations on

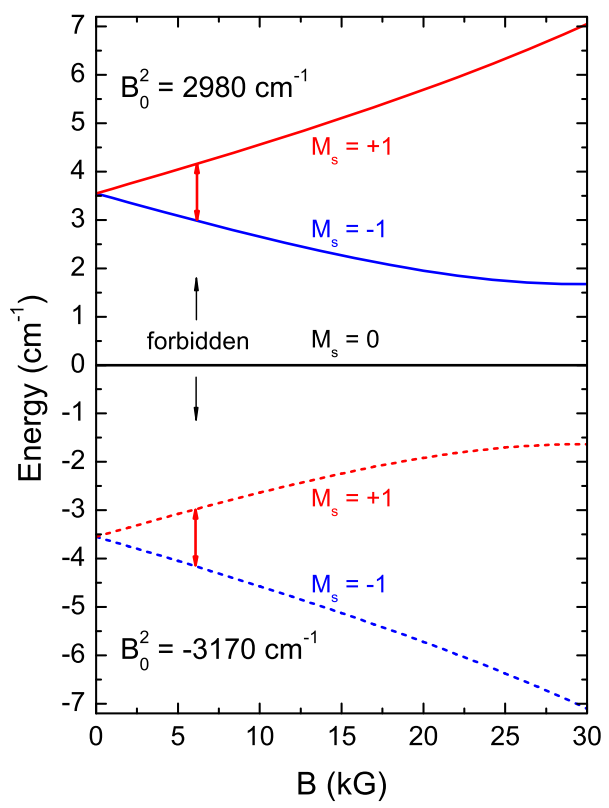

Figure 8 Magnetic field dependence for $\varangle(B, c)=20^{\circ}$ of the ground state energy levels for the $\mathrm{Ni}^{2+}\left(\mathrm{d}^{8}\right)$ system calculated with the program CONCORD using the Wybourne parameters $B_{0}^{4}=-17,300 \mathrm{~cm}^{-1}, \quad B_{3}^{4}=20,700 \mathrm{~cm}^{-1}$ for two different trigonal parameters $B_{0}^{2}$ given in the figure. The curve with $\mathrm{M}_{\mathrm{S}}=0$ belongs to both sets of ground state levels. The forbidden $\left(\left|\Delta \mathrm{M}_{\mathrm{S}}\right|=2\right)$ transitions in the $Q$-band $(34.0 \mathrm{GHz})$ are marked by arrows.

$\mathrm{Cr}^{3+}(S=3 / 2)$ and $\mathrm{Fe}^{3+}(S=5 / 2)$ doped hexagonal $\mathrm{BaTiO}_{3}$ that paramagnetic ions located both at $\mathrm{Ti}(1)$ and $\operatorname{Ti}(2)$ sites have different $\mathrm{D}$ values and also different signal intensities, i.e., different incorporation probabilities for site $\mathrm{Ti}(1)$ or $\mathrm{Ti}(2)$. This information can in principle be used to identify the site related to the different EPR spectra [29, 39]. In the present case, however, unfortunately only one very broad line is observed. Moreover, it is possible that the different influence of the oxygen surroundings of the Ti(1) and $\mathrm{Ti}(2)$ sites on the D value is small compared to its very high value of $3.55 \mathrm{~cm}^{-1}$. Hence, the observed broad line could be caused by overlapping signals of $\mathrm{Ni}^{2+}$ ions located at both positions $\mathrm{Ti}(1)$ and $\mathrm{Ti}(2)$.

The capability of the program CONCORD to also fit eigenvalue differences $\Delta \mathrm{E}$ between energetically lowest microstates can be used to fit the experimental $D$ value. On the other hand, in the case of a trigonal crystal field with three parameters $B_{0}^{4}, B_{0}^{2}$ and $B_{3}^{4}$ the system is underdetermined if only one experimental parameter $\mathrm{D}$ is available. Hence, we tried to fit the crystal field parameters using both D and the $\chi_{m o l}(\mathrm{~T})$ data simultaneously. This procedure becomes 
possible by applying the advanced nonlinear fitting tool of the program ORIGIN [40] in co-operation with CONCORD. In the case of $\mathrm{Ni}^{2+}$ ions, the dependence of $\chi_{\mathrm{mol}}(\mathrm{T})$ on the parameters $B_{0}^{2}$ and $B_{3}^{4}$ is quite weak as discussed in "Magnetic susceptibility of ceramic samples" section. For this reason, a successful simultaneous fit was only possible upon fixing of $B_{0}^{4}$ to the value of $-17,300 \mathrm{~cm}^{-1}$ used above in the fitting of the $\chi_{\mathrm{mol}}(\mathrm{T})$ data with a cubic crystal field. The resulting trigonal crystal field parameters amount to $B_{0}^{2} \approx 2500 \mathrm{~cm}^{-1}$ and $B_{3}^{4} \approx 19,000 \mathrm{~cm}^{-1}$ with an uncertainty of about $30 \%$. On the other hand, the fit of the experimental data of $\mathrm{D}$ and $\chi_{\mathrm{mol}}(\mathrm{T})$ is also possible using a negative value of $B_{0}^{2}$ of the same magnitude. Hence, the determination of the correct incorporation site $(\mathrm{Ti}(1)$ or $\operatorname{Ti}(2))$ is actually not possible.

\section{Conclusions}

Temperature-dependent measurements of the molar paramagnetic susceptibility of Ni-doped hexagonal $6 \mathrm{H}-\mathrm{BaTiO}_{3}$ ceramics $(0.5-2.0 \mathrm{~mol} \% \mathrm{Ni})$ sintered in air at $1400{ }^{\circ} \mathrm{C}$ prove that the majority of the $\mathrm{Ni}$ ions is in the $2+$ valence state. At $\mathrm{Ni}$ concentrations of $\geq 1.5$ $\mathrm{mol} \%$, a weak antiferromagnetic interaction between the $\mathrm{Ni}^{2+}$ ions is found. Room-temperature, angledependent EPR Q-band measurements on a $2.0 \mathrm{~mol} \%$ Ni-doped single crystal revealed an associate between $\mathrm{Ni}^{3+}$ and a neighboring oxygen vacancy. Comparing the directions of the main axes of the gtensor of this associate with the directions of the Ti-O bonds in the hexagonal $\mathrm{BaTiO}_{3}$ structure, oxygen vacancies at the $\mathrm{O}(1)$ site can be ruled out. Moreover, the existence of additional $\mathrm{Ni}^{3+}$ ions, which are not associated with oxygen vacancies and located at $\mathrm{Ti}(1)$ or $\mathrm{Ti}(2)$ sites, was confirmed. This already known defect type [23] was identified by its isotropic gvalue. Finally, angle-dependent EPR single-crystal measurements also revealed a further $\mathrm{Ni}^{2+}$ defect, which could not be observed in previous investigations on ceramic samples [23]. Due to its very high ZFS of $3.55 \mathrm{~cm}^{-1}$, only the forbidden transition $\Delta M_{S}= \pm 2$, which possesses a low signal intensity, can be observed. Because of the angle-dependence peculiarities for this forbidden transition, its broad line could be detected only for angles between the static magnetic field and the $c$ axis in the range between $20^{\circ}$ and $75^{\circ}$. A simultaneous fit of the temperature-dependent susceptibility and the EPR fine structure parameter $\mathrm{D}$ of the $\mathrm{Ni}^{2+}$ spectrum allowed the estimation of the parameters of the trigonal crystal field to $B_{0}^{4} \approx-17,300 \mathrm{~cm}^{-1},\left|B_{0}^{2}\right| \approx 2500 \mathrm{~cm}^{-1}$ and $B_{3}^{4} \approx 19,000 \mathrm{~cm}^{-1}$. Since only one and rather broad $\mathrm{Ni}^{2+}$ line could be detected, no information on the location of the $\mathrm{Ni}^{2+}$ ion $(\mathrm{Ti}(1)$ or $\mathrm{Ti}(2))$ can be given. It is also possible that the line is caused by nickel ions positioned on both sites.

\section{Acknowledgement}

The authors thank Christine Seidel (Institute of Chemistry, Faculty of Natural Sciences II, MartinLuther-Universität Halle-Wittenberg) for the laborious preparation of tiny single-crystalline samples of sufficient crystalline quality.

\section{Funding}

Open Access funding enabled and organized by Projekt DEAL.

\section{Compliance with ethical standards}

Conflict of interest The authors declare that they have no conflict of interest.

Open Access This article is licensed under a Creative Commons Attribution 4.0 International License, which permits use, sharing, adaptation, distribution and reproduction in any medium or format, as long as you give appropriate credit to the original author(s) and the source, provide a link to the Creative Commons licence, and indicate if changes were made. The images or other third party material in this article are included in the article's Creative Commons licence, unless indicated otherwise in a credit line to the material. If material is not included in the article's Creative Commons licence and your intended use is not permitted by statutory regulation or exceeds the permitted use, you will need to obtain permission directly from the copyright holder. To view a copy of this licence, visit http://creativecommons.org/licen ses/by/4.0/. 


\section{References}

[1] Ray S, Mahadevan P, Mandal S, Krishanakumar RS, Kuroda CS, Sasaki T, Taniyama T, Itoh M (2008) High temperature ferromagnetism in single crystalline dilute Fe-doped $\mathrm{BaTiO}_{3}$. Phys Rev B 77:104416

[2] Chakraborty T, Ray S, Itoh M (2011) Defect-induced magnetism: test of dilute magnetism in Fe-doped hexagonal $\mathrm{BaTiO}_{3}$ single crystals. Phys Rev B 83:144407

[3] Valant M, Arčon I, Mikulska I, Lisjak D (2013) Cation order-disorder transition in Fe-doped $6 \mathrm{H}-\mathrm{BaTiO}_{3}$ for dilute room-temperature ferromagnetism. Chem Mater 25:3544-3550

[4] Zorko A, Pregelj M, Gomilšek M, Jagličić Z, Pajić D, Telling M, Arčon I, Mikulska I, Valant M (2015) Straininduced extrinsic high-temperature ferromagnetism in the Fe-doped hexagonal barium titanate. Sci Rep 5:7703

[5] Prellier W, Fouchet A, Mercey B, B, (2003) A ten-year perspective on dilute magnetic semiconductors and oxides. J PhysCondens Matter 15:R1583-R1601

[6] Dietl T (2010) Oxide-diluted magnetic semiconductors: a review of the experimental status. Nat Mater 9:965-974

[7] Curecheriu L, Postolache P, Buscaglia MT, Buscaglia V, Ianculescu A, Mitoseriu L (2014) Novel magnetoelectric ceramic composites by control of the interface reactions in $\mathrm{Fe}_{2} \mathrm{O}_{3} @ \mathrm{BaTiO}_{3} \quad$ core-shell structures. J ApplPhys 116:084102

[8] Rajan S, Mohammed Gazalli PM, Okrasa L, Chandrasekaran $\mathrm{G}$ (2018) Multiferroic and magneto-dielectric properties in $\mathrm{Fe}$ doped $\mathrm{BaTiO}_{3}$. J Mater Sci Mater Electron 29:11215-11228

[9] Barbier A, Aghavnian T, Badjeck V, Mocuta C, Stanescu D, Magnan H, Rountree CL, Belkhou R, Ohresser P, Jedrecy N (2015) Antiferromagnetic long-range spin ordering in Feand $\mathrm{NiFe}_{2}$-doped $\mathrm{BaTiO}_{3}$ multiferroic layers. Phys Rev B 91:035417

[10] Das SK, Mishra RN, Roul BK (2014) Magnetic and ferroelectric properties of Ni doped $\mathrm{BaTiO}_{3}$. Solid State Commun 191:19-24

[11] Madhan K, Murugaraj R (2020) Structural, electrical, and weak ferromagnetic-to-antiferromagnetic nature of $\mathrm{Ni}$ and $\mathrm{La}$ co-doped $\mathrm{BaTiO}_{3}$ by sol-gel combustion route. J Sol-Gel SciTechn 95:11-21

[12] Rečnik A, Kolar D (1996) Exaggerated growth of hexagonal barium titanate under reducing sintering conditions. J Am Ceram Soc 79:1015-1018

[13] Glaister RM, Kay HF (1960) An investigation of the cubichexagonal transition in barium titanate. ProcPhysSoc $76: 763-771$
[14] Keith GM, Rampling MJ, Sarma K, Alford NM, Sinclair DC (2004) Synthesis and characterisation of doped $6 \mathrm{H}-\mathrm{BaTiO}_{3}$ ceramics. J Euro Ceram Soc 24:1721-1724

[15] Jayanthi S, Kutty TRN (2008) Dielectric properties of 3d transition metal substituted $\mathrm{BaTiO}_{3}$ ceramics containing the hexagonal phase formation. J Mater Sci Mater Electron 19:615-626

[16] Shiozaki Y, Nakamura E, Mitsui T (Eds.) (2001) LandoltBörnstein New series III/36A1 Ferroelectrics and related substances_oxides. Springer Materials, https://doi.org/10.1 007/b53034

[17] Noda Y, Akiyama K, Shobu T, Kuroiwa Y, Yamaguchi H (1999) High-resolution powder diffractometry to study the phase transition of h-BaTiO 3 . Jpn J ApplPhys 38:73-76

[18] Burbank RD, Evans HT Jr (1948) The crystal structure of hexagonal barium titanate. ActaCrystallogr 1:330-336

[19] Hagemann H-J, Ihrig H (1979) Valence change and phase stability of 3d-doped $\mathrm{BaTiO}_{3}$ annealed in oxygen and hydrogen. Phys Rev 20:3871-3878

[20] Hagemann H-J, Hennings D (1981) Reversible weight change of acceptor-doped $\mathrm{BaTiO}_{3}$. J Am Ceram Soc 64:590-594

[21] Langhammer HT, Müller T, Felgner K-H, Abicht H-P (2000) Influence of strontium on manganese-doped barium titanate ceramics. Mat Lett 42:21-24

[22] Desu SB, Subbarao EC (1980) Inhibition of reduction of $\mathrm{BaTiO}_{3}$. J Mat Sci 15:2113-2115

[23] Böttcher R, Langhammer HT, Müller T (2011) Paramagnetic resonance study of nickel ions in hexagonal barium titanate. J PhysCondens Matter 23:115903

[24] Lenjer S, Scharfschwerdt R, Kool ThW, Schirmer OF (2000) An off-center ion near a $\mathrm{Ba}$ site in $\mathrm{BaTiO}_{3}$ as studied by EPR under uniaxial stress. Solid State Commun 116:133-136

[25] Kool ThW, Lenjer S, Schirmer OF (2007) Jahn-Teller and off-center defects in $\mathrm{BaTiO}_{3}: \mathrm{Ni}^{+}, \mathrm{Rh}^{2+}, \mathrm{Pt}^{3+}$ and $\mathrm{Fe}^{5+}$ as studied by EPR under uniaxial stress. J PhysCondens Matter $19: 496214$

[26] Fu C, Chen N, Du G (2017) Comparative studies of nickel doping effects at $\mathrm{A}$ and $\mathrm{B}$ sites of $\mathrm{BaTiO}_{3}$ ceramics on their crystal structures and dielectric and ferroelectricproperties. Ceram Internat 43:15927-15931

[27] Lebedev AI, Sluchinskaya IA (2016) On the nature of change in $\mathrm{Ni}$ oxidation state in $\mathrm{BaTiO}_{3}-\mathrm{SrTiO}_{3}$ system. Ferroelectrics 501:1-8

[28] Dawson JA, Freeman CL, Harding JH, Sinclair DC (2013) Phase stabilisation of hexagonal barium titanate doped with transition metals: a computational study. J Solid State Chem 200:310-316

[29] Böttcher R, Langhammer HT, Kücker S, Eisenschmidt C, Ebbinghaus SG (2018) On the incorporation of iron into 
hexagonal barium titanate: I. Electron paramagnetic resonance (EPR) study. J PhysCondens Matter 30:425701

[30] Langhammer HT, Walther T, Böttcher R, Ebbinghaus SG (2020) On the incorporationof iron into hexagonal barium titanate: II. Magnetic moment, electron paramagnetic resonance (EPR) and optical transmission. J PhysCondens Matter 32:385702

[31] Stoll S (2015) CW-EPR spectral simulations: solid state. Methods Enzymol 563:121-142

[32] Abragam A, Bleaney B (1970) Electron paramagnetic resonance of transition ions. Clarendon, Oxford

[33] Lueken H (1999) Magnetochemie. Teubner, Stuttgart

[34] Hatscher S, Schilder H, Lueken H, Urland W (2005) Practical guide to measurement and interpretation of magnetic properties. Pure ApplChem 77:497-511

[35] Schilder H (2015) Programm CONCORD. Fachhochschule Aachen http://www.condon.fh-aachen.de
[36] Langhammer HT, Böttcher R, Müller T, Walther T, Ebbinghaus SG (2015) Defect properties of cobalt-doped hexagonal barium titanate ceramics. J PhysCondens Matter 27:295901

[37] Wybourne BG (1965) Spectroscopic properties of rare earths. Wiley, New York

[38] Akimoto J, Gotoh Y, Oosawa Y (1994) Refinement of hexagonal $\mathrm{BaTiO}_{3}$. ActaCrystallogr C 50:160-161

[39] Langhammer HT, Müller T, Böttcher R, Abicht H-P (2008) Structural and optical properties of chromium-doped hexagonal barium titanate ceramics. J PhysCondens Matter 20:085206

[40] OriginPro, Version 7.5 SR7, OriginLab Corporation, Northampton, MA, USA

Publisher's Note Springer Nature remains neutral with regard to jurisdictional claims in published maps and institutional affiliations. 